Physics, Chemistry, and Dynamics of Interplanetary Dust

ASP Conference Series, Vol. 104, 1996

Bo A. S. Gustafson and Martha S. Hanner (eds.)

\title{
SUMMARY REMARKS
}

\author{
Philippe L. Lamy
}

Laboratoire d'Astronomie Spatiale, BP 8, F-13376 Marseille Cedex 12

\section{Introduction}

The excellent health of our discipline is well illustrated not only by the global statistics of this colloquium ( $\sim 75$ oral presentations and $\sim 40$ posters) but, more important, by the report of truly new "discoveries" and by the continuous progress and refinement of the analysis.

One striking feature is the continuous evolution of the analysis of the Zodiacal Cloud, not as a single entity, but as a superposition of components which are directly tied to their origin:

- asteroidal

- cometary

- interstellar

- jovian dust streams

- planetary rings

- space debris

and whose relative importance depends upon the location in the solar system. The contributions of these various sources may be either to the global cloud or to its distinct structures or both: this is particularly the case of asteroids (with the asteroidal dust bands) and comets (with the cometary dust trails and the meteoroid streams). Of course, the very perception of structures is tied to a particular view of the solar system at a particular time; structures may eventually dilute and disappear in the general interplanetary background.

All major lines of investigation, remote observations (visible, infrared), insitu space detections and laboratory analysis, now aim and are more or less able to distinguish all or part of these components. This very parallel approach, which must be pursued, is highly fruitful and obviously a source of mutual progress.

Let us not forget that the meteor data had already revealed several of those components and I certainly want to acknowledge the contribution of Ceplecha here. I very much regret that those data have not been incorporated in the recent attempts of "global" analysis. The fieid of radio-meteors is still very active and new technical developments are under way to increase the sensitivity and the accuracy of the measurements. It will therefore remain an important source of information, of improved quality, on the IDP's. 


\section{Important new results}

Let me now come to several important new results since the last meeting (Kyoto, Japan, 1990), namely the detection of interstellar grains, of Jovian dust streams and of a circum-solar dust ring ("the Earth resonance ring").

\subsection{Interstellar grains}

Interstellar grains have been isolated in IDP's (Gems, diamonds) and are available in the laboratory as fossil evidences of the early times of the solar system. Their penetration in the present solar system has been predicted and studied but from a rather academic point-of-view. Their actual detection has now been achieved by several means of investigation. The grains have been unambiguously detected in the solar system by Ulysses, Galileo and possibly, by the Hiten spacecraft.

Of course, the most thriving aspect is the possibility now open to collect them and that has not escaped the attention of our colleagues proposing the "Stardust" mission. This further opens the possibility to compare "fresh" individual interstellar grains with those preserved in IDP's and meteorites.

\subsection{Jovian dust streams}

Jovian dust streams have been detected by the Ulysses spacecraft but their exact origin remains elusive. This hopefully will be uncovered by the Galileo space craft in the coming months. The next question will be: Do Saturnian streams exist? The Cosmic Dust Analyzer on Cassini will have to watch for them.

\subsection{The Earth resonance ring}

The elusive circum-solar dust ring has at last been detected but not where it was expected. We searched between 3 and $20 \mathrm{R}_{\odot}$, it is at 1 AU! Very surprisingly, the observational evidences (i.e., the leading/trailing asymmetry in the Zodiacal Light) were available a long time ago in Dumont's data, Leinert's data and probably others as well. They never really retained attention and were even considered as artifact (e.g., in the analysis of the IRAS data). A combination of fine analysis and superb dynamical modeling have led to the discovery of this ring which, in my opinion, could be named the "Earth resonance ring" to avoid ambiguities with the classical circum-solar dust rings.

Regarding the "true" circum-solar ring, three independent observations during the 1991 (Hawaii) eclipse have convincingly given a negative answer; no ring structure could be detected. There are various attempts to explain reported detections at other times by variable phenomenae. In my opinion, a permanent, conspicuous structure is improbable as the Zodiacal Cloud progressively collapses near the Sun. The only large dusty transitory effects are Sun-grazing comets. As the remnants can appear as two patches on either sides of the Sun, some past positive results may have just witnessed such an event.

I just want to conclude this first part by underlining the tremendous potential of the Ulysses, Galileo, Hiten and COBE data which are far from having produced all the science they intrinsically contain. It is self-evident that they are unique data benefiting from improved technology, i.e., improved sensitivity. Let me suggest two potentially promising lines of investigation: 

data

i) the study of $\beta$-meteoroids, whose evidence is still meager, using the Hiten

ii) the in-depth analysis of the Zodiacal Light combining the COBE visible (+ polarization) and infrared (thermal) data. It is just too bad that they do not cover the full ecliptic sky.

\section{Laboratory analysis of IDP's}

Once again, the groups performing laboratory analysis have amazed us by their increasing and endless capability in analyzing IDP's at incredible scales with incredible refinement. This set of contributions was one of the highlights of this colloquium in terms of both the quality of the results and the quality of their presentations. In addition to the new findings on the "classical" stratospheric IDP's, this is the first time that we see extended and very valuable results from another source, the polar ices IDP's. With 100,000 of them and the presence of (almost) unaltered materials up to a size of $1 \mathrm{~mm}$, we have an unprecedented increase in the quantity and diversity of extraterrestrial material available in the laboratory. Let me emphasize several selected results:

i) the classification scheme of the IDP's is evolving in the sense that all of them are now characterized as chondritic, i.e., a chondritic composition within a factor 2, although Jessberger et al. distinguish two additional groups (low $\mathrm{Zn}$ and low $\mathrm{Ni}$ )

ii) the $\mathrm{Si} / \mathrm{Mg}$ ratio remains approximately constant while the $\mathrm{Fe}$ content varies among IDP's

iii) Carbon is amorphous and its content ranges from 5 to $25 \%$ (higher than in meteorites); it is found in the form of chunks and even as a matrix of Gem's but not in the coating of the IDP's

iv) Calcium is depleted, a fact which may be connected to the presence of carbonates

v) volatile trace elements are enriched by factors of 2 to 5 except for Sulfur (similar to CI) and Bromine for which possible contamination effects have been noted

vi) the organic material is different from that found in meteorites.

Altogether there are growing evidences that IDP's are a very distinct class from the (CI) meteorites and Flynn et al. have suggested that they may come from the last state of condensation of the solar nebula (later than the CI's).

The origin of the IDP's have been delineated to some extent, in connection to their porosity and to their reflectivity spectra

- asteroidal (mostly main belt asteroids)

- cometary and asteroidal from distant, S-type asteroids (the distinction is difficult)

- interstellar 
The study of the Gem's reported by Bradley has been, in my opinion, a stunning moment of this conference. In summary, their main properties are:

- a chondritic composition but depleted in Sulfur and Magnesium

- a segregation of the Magnesium which is preferentially found at their surface, possibly a result of prior irradiation

- a structure, composed of FeS or Fe and a mantle of amorphous silicate depleted in Sulfur, derived from interstellar grain cores

However there also exists another class of Gem's with different properties: they are embedded in a carbon matrix and show no segregation of the Magnesium.

\section{Light scattering}

Laboratory works are in progress both in the microwave domain here at the University of Florida and in the visible for which two experiments have been reported. Both approaches have their advantages and drawbacks and must be pursued in parallel. Detailed experimental results are indeed required to feed much needed theoretical developments on the light scattering by complex, irregular particles, a domain for which significant progress have not been reported. The discrete dipole approximation (DDA) is very appealing because it is easily implemented on computers. Memory space limits the total number of dipoles so that large particles cannot be handled. Some limited extensions to circumvent the intrinsic limitations of the DDA are of questionable validity. For instance, the introduction of mixing rules (e.g., Maxwell-Garnett) is not satisfactory at all to handle inhomogeneous particles. Altogether, an effort of clarification of the available methods is required and imaginative, new approaches are badly needed.

For the experts in the field, I would like to call attention to a contribution on coherent backscattering by Horn et al. This has to do with a fundamental optical process known as the localization principle which, I think, has also been seen in the Gegenschein.

\section{Planetary rings}

The most interesting dynamical evolution of dust particles probably takes place in planetary rings as a variety of forces are at work. They represent indeed a very good case where electromagnetic forces are significant, the best example being offered by the role of Lorentz resonance in the Jovian ring. Although the field has witnessed tremendous development in the past years leading to the specific terminology of "dusty plasma", there are still important aspects which are poorly or not even understood, e.g..

- the clumpiness of certain rings

- the replenishment, i.e., the sources that supply sufficient amounts of dust to maintain those rings that have very short lifetimes 


\section{Dynamics}

Nature has been sweet to us in the sense that one can distinguish two situations depending upon whether or not the electromagnetic forces are important, situations which depend upon the size of the dust particles.

i) Large particles

The most important processes are:

- gravity

- radiation pressure force

- dissipative forces (Poynting-Robertson drag)

- collisions

- planetary perturbations.

The importance of the latter process has been dramatically demonstrated by the work of Liou et al. which reveals that the time scale of the dynamical evolution of IDP's may not be dominated by their Poynting-Robertson lifetimes but by the time they get trapped into a planetary resonance.

ii) Small particles

This concerns particularly the interstellar grains penetrating the solar system for which the dominating process is the Lorentz force. Clearly, we do not have to worry about the planetary resonances or drag and probably not about collisions.

A striking event of the past years has been the eruption of massive dynamical calculations with super-computers. One of the first work (at least the first which came to my attention) was by Jackson \& Zook (1992) which clearly demonstrated the role of planetary perturbation and specifically, resonances (an earlier but limited work by Gonczi et al. (1982) may be noted). Numerical calculations are now widespread and the group here at the University of Florida has been very active and have offered a beautiful application with the Earth resonance ring.

The study of meteor streams has also enormously benefited from extensive numerical calculations leading to predict both past and future shower activity, particularly meteor storms (or bursts). However., I noted possible conflicting interpretations of these bursts in terms of the intrinsic activity of the parent comet or in terms of the location of the Earth in an inhomogeneous, structured stream (are both interpretations equally valid depending upon the case?).

In the midst of all these computer calculations, is there still room for analytical investigations? Certainly so and recently Richter \& Keller (1995) offered a new analytical solution for the case of dust particles orbiting a cometary nucleus. Even if this kind of "new" solution is not discovered every day, we have seen at this conference niches where analytical studies are valuable and moreover, enlightening. A few examples are: 
- local treatment to better study a resonance

- discovery of new integrals of complex motion (Gor'kavyi et al.)

- "hybrid" treatment as proposed by Hamilton where perturbations are numerically averaged

\section{Cometary dust}

Although cometary dust is a bit outside our main field, I wish to point out the very interesting detailed analysis of the most prominent bands seen in comets:

i) the $3.4 \mu \mathrm{m}$ band in terms of several components

ii) the 10 and $20 \mu \mathrm{m}$ silicate bands in relationship with

- the presence of crystalline olivine

- the olivine/pyroxene ratio

- the $\mathrm{Fe} / \mathrm{Mg}$ and $\mathrm{Si} / \mathrm{Mg}$ ratios

Further comparisons with the $\beta$-pictoris ring and other circum-stellar objects is also of great interest.

Another interesting aspect is the fragmentation of dust grains in comae, for which there is growing evidences. This process is possibly connected to the diffuse source of several species, notably CO.

\section{Toward a global view}

The classical question of the connection of IDP's, asteroidal versus cometary, should first be broadened to include other components and second be generalized by realizing that the answer depends upon the location of the observer in the solar system and upon what he observes or detects. Regarding the flux number of IDP's at $1 \mathrm{AU}$, Dermott et al. found that $70 \%$ comes from comets and $30 \%$ from asteroids, whereof $5 \%$ are from specific asteroid families. Once again, this is the situation that prevails at $1 \mathrm{AU}$ and note that the picture will be entirely different at other places in the solar system. Hughes worked out the mass budget here again at $1 \mathrm{AU}$ and concluded that it is dominated by dust of cometary origin although the present values are highly uncertain.

A more general or global approach called "full component analysis" is proposed in Figure 1. It presents distinct differences from that implemented by Divine (1993) in the sense that it avoids introducing arbitrary dust populations while incorporating as much physical constraints as possible. It has in fact already been implemented in a few specific cases. Here, I propose a systematic analysis by physical components (asteroidal, cometary, interstellar ...) followed by a synthesis aimed at investigating global questions. For each component, the properties of the dust particles are inferred from available observations and, if possible, derived from laboratory analysis of IDP's. The dynamical evolution is then investigated taking into account the appropriate origin (asteroid, comet...) 
Component

(asteroid,comet, interstellar....)

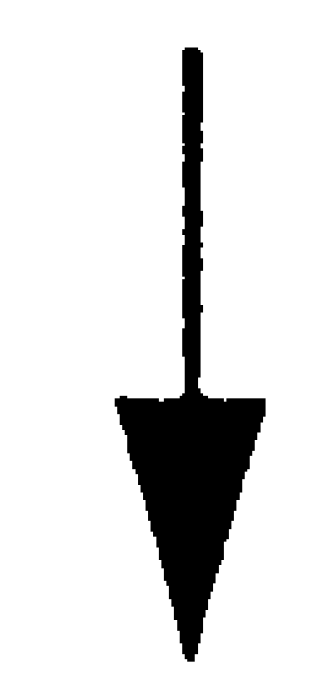

Properties of dust particles

bulk density

temperature

$\beta$

charge
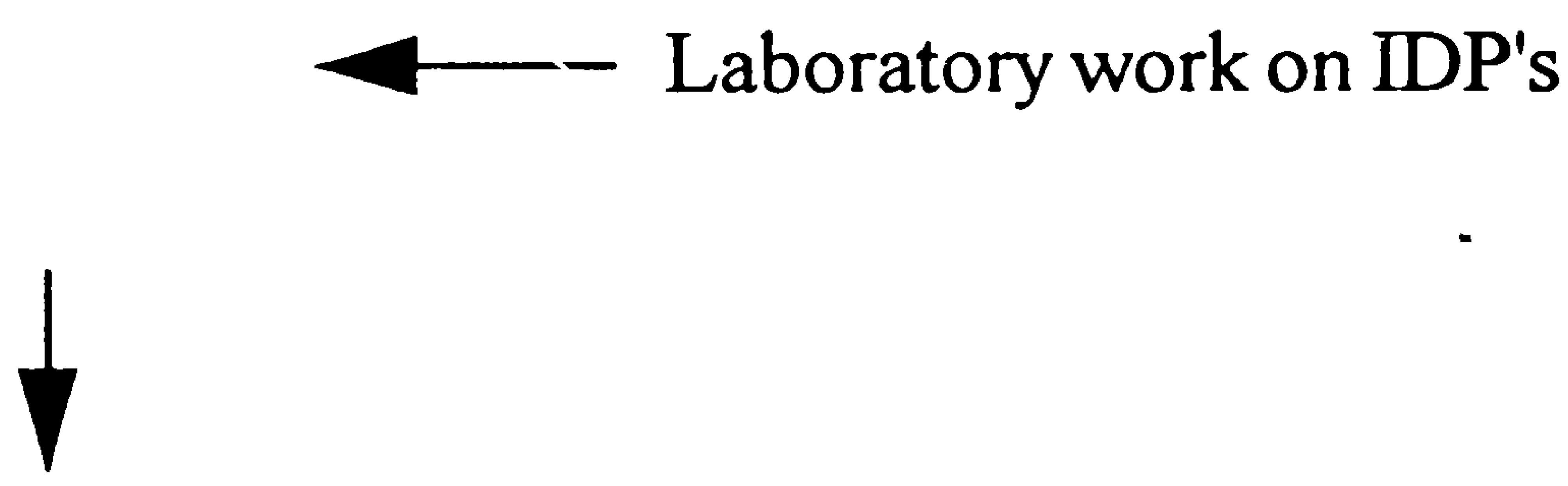

Dynamics of dust particles

1

PredictFlux/Spatial density

$\mathrm{F}(\mathrm{R}, \theta, \varphi$, size $)$

$\downarrow$

Confront to observations

1

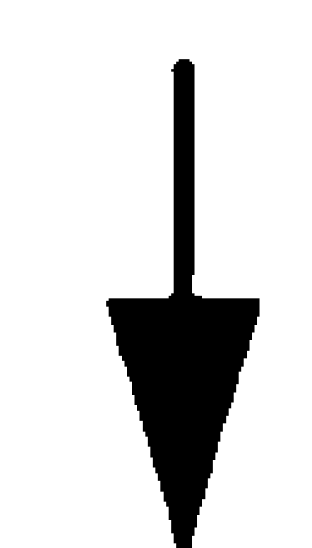

1

1

In-situ (impacts) Remote Radar Collections

Earth

VIS

Moon

IR

Space Probes

Compare $\Sigma \alpha_{\mathfrak{t}}$ component $_{\mathrm{i}}$ with observations

Study time variations $\left(0.4 \rightarrow 10^{8} \mathrm{y}\right)$

Figure 1. Proposed full component analysis 
and the differential spatial flux and density are calculated at a specific location of interest in the solar system. The results are confronted to all available observational evidences at the same location. Other questions, such as the time variations may be studied as well. The synthesis involved a summation over all components with scaling factors and again, a comparison with observations.

\section{The Future of our field}

The future of our field looks to me extremely bright with first a handful of space missions, either on-going or to be launched, which are all able to bring new data on the Zodiacal Cloud complex thanks to both remote and in-situ observations. The list includes Galileo, the extended Ulysses mission, COBE, Eureca ISO, SOHO, Cassini, Planet-B, Rosetta and Stardust. In the case of the Cassini mission, it is of utmost importance that cruise science be implemented so as to probe a vast extent of interplanetary space with new, highly sensitive detectors. Possible new missions could include:

i) the collection of interstellar dust

ii) the observation of the inner zodiacal light and F-corona on a Moon orbiter (similar to Clementine but with improved, properly calibrated cameras).

Collections, stratospheric and polar ices, are actively going on as well as laboratory works on the analysis of IDP's and more generally, on laboratory astrophysics. Super-computers will allow tremendous developments of dynamical calculations. Laboratory studies of the light scattering, both in the microwave and optical domains, are proceeding extremely well. But here again, and to conclude, I wish to emphasize the need for innovative approaches to the problem of light scattering by complex particles.

Acknowledgments. I wish to thank the SOC and Martha Hanner in particular for their invitation to give this summary talk.

\section{References}

All quoted references may be found in this volume except:

Divine N. 1993, Five populations of interplanetary meteoroids, J. Geophys. Research, 98, 17,029-17,048.

Gonczi R., Ch. Froeschle \& Cl. Froeschle 1982, Poynting-Robertson drag and orbital resonance, Icarus, 51, 633-654.

Jackson A.A., \& H.A. Zook 1992, Orbital evolution of dust particles from comets and asteroids, Icarus, 97, 70-84.

Richter K., \& H.U. Keller 1995, On the stability of dust particle orbits around cometary nuclei, Icarus, 114, 355-371. 